\title{
Fluorometric Viral miRNA Nanosensor for Diagnosis of Productive (Lytic) Human Cytomegalovirus Infection in Living Cells
}

\author{
Ji-Seon Lee, ${ }^{\text {a }}$ Seongchan Kim, ${ }^{\text {a }}$ Sungchul Kim,,${ }^{\text {b,c }}$ Kwangseog Ahn ${ }^{\text {b,c }}$ and Dal-Hee Min*,a,b,d \\ ${ }^{a}$ Department of Chemistry, Seoul National University, Seoul, 08826, Republic of Korea \\ ${ }^{\mathrm{b}}$ School of Biological Sciences, Seoul National University, Seoul 08826, Republic of Korea \\ ${ }^{\mathrm{c}}$ Center for RNA Research, Institute for Basic Science (IBS), Seoul, 08826, Republic of Korea \\ ${ }^{d}$ Institute of Biotherapeutics Convergence Technology, Lemonex Inc., Seoul, 08826, Republic of Korea
}

\section{Present Addresses}

Center for Biomaterials, Biomedical Research Institute, Korea Institute of Science and Technology (KIST), 5 Hwarangro 14-gil, Seongbuk-gu, Seoul 02792, Republic of Korea (Seongchan Kim).

Kavli Institute of Nanoscience, Department of Bionanoscience, Delft University of Technology, Delft 2628, The Netherlands (Sungchul Kim). 


\section{Experimental Section}

Preparation of a dextran-coated graphene oxide nanocolloid (DGON). Potassium persulfate ( $4 \mathrm{~g}$, Junsei Chemical Co., Japan) and phosphorus pentoxide (4 g, Junsei Chemical Co., Japan) were added to $50 \mathrm{~mL}$ of sulfuric acid (Samchun, Korea), and $2.0 \mathrm{~g}$ of graphite nanofiber ( $300 \mathrm{~nm}$ in diameter, $30 \mu \mathrm{m}$ in length, Carbon Nanomaterial Technology, Korea) was added to the prepared acid mixture and stirred at $90^{\circ} \mathrm{C}$ or $16 \mathrm{~h}$. The prepared pre-oxidized graphite nanofiber was added to 250 $\mathrm{mL}$ of sulfuric acid in an ice bath. $10 \mathrm{~g}$ of potassium permanganate (Aldrich Chemical Co., USA) was slowly added to the mixture and then, transferred to $35^{\circ} \mathrm{C}$ water bath and stirred for $6 \mathrm{~h}$. After dilution and cooling down, $50 \mathrm{~mL}$ of hydrogen peroxide ( $30 \%$, Junsei Chemical Co., Japan) was added to the mixture and the mixture was centrifuged at 10,000 rpm for 30 min and rinsed with 3.4\% hydrochloric acid (Samchun, Korea), acetone repeatedly. The supernatant of acetone was collected and evaporated under vacuum. The collected solid was dispersed in water and dialyzed with $10 \mathrm{kDa}$ membrane tube until the suspension was neutralized. The purified suspension was lyophilized to collect graphene oxide nanocolloid powder. Finally, $50 \mathrm{mg}$ of graphene oxide nanocolloid dispersed in $50 \mathrm{~mL}$ of $0.1 \%$ aqueous dextran $(\sim 6 \mathrm{kDa}$, from Leuconostoc ssp., Fluka, USA) solution was mixed with $25 \mu 1$ of ammonium hydroxide ( $28 \sim 30 \%$ in $\mathrm{H} 2 \mathrm{O}$, Sigma-Aldrich, USA) and heated at $95^{\circ} \mathrm{C}$ for $3 \mathrm{~h}$ with stirring. Then, the prepared material was collected by repeated centrifugation and purified with dialysis membrane tube.

Characterization of the DGON. UV-Vis absorbance and fluorescence spectra were obtained by using UV-Vis spectrophotometer S-3100 (Scinco, Seoul, Korea) and fluorometer SynergyMx (Biotek, UK), respectively. Atomic force microscopy (AFM) image with height profile were analyzed by NTEGRA Spectra II \& Nova Px software (NT-MDT Spectrum Instruments, Russia). The measurement of FT-IR spectra was performed with Vertex70 FT-IR spectrometer equipped with HYPERION 1000 microscope (Bruker, USA). Zeta potential and dynamic light scattering (DLS) analysis were performed by using Zetasizer NanoS (Malvern instruments, UK). Raman spectra were measured by LabRAM HR800 micro-Raman spectrometer (Horiba Jobin Yvon, USA) using an Ar ion CW laser $(514.5 \mathrm{~nm})$ as an excitation source focused through a BXFM confocal microscope with an objective 50x lens.

Cell Culture. Human foreskin fibroblast (HFF) cells were cultured in DMEM containing D-glucose (4.5 g/L) and supplemented with $10 \%$ FBS, penicillin $(100$ units $/ \mathrm{mL})$, and streptomycin $(100 \mathrm{~g} / \mathrm{mL})$. The cells were maintained in a humidified incubator under $5 \% \mathrm{CO} 2$ at $37^{\circ} \mathrm{C}$.

Cell viability assay with DGON. To examine the cytotoxicity of the DGON, cell viability was estimated by using cell counting kit-8 (CCK-8) after treatment of the DGON. HFF cells were seeded in a 96-well cell culture plate at a density of 1.4 
$\times 10^{4}$ cells per well in culture media and incubated for $24 \mathrm{~h}$. Then, the cells were treated with varying concentrations of the DGON in complete medium. After $12 \mathrm{~h}$ of treatment, media was removed and washed with PBS, followed by further incubation with a mixture of serum-free fresh media $(100 \mu \mathrm{L})$ and CCK-8 solution $(10 \mu \mathrm{L})$ for $2 \mathrm{~h}$. Then, absorbance at 460 and $670 \mathrm{~nm}$ was measured by using a microplate reader (Molecular Devices Inc., Sunnyvale, CA, USA). All experiments were performed in triplicate.

Titration of HCMV used in this study. To determine the titer of the HCMV Townelong strain used in this research, Immunofluorescence assay was performed according to the following method (Figure S6). HFF cells were seeded onto the cover glass in 12-well culture plate with a density of $2.0 \times 10^{4}$ cells per well for $24 \mathrm{~h}$. The diluted HCMV viral samples (dilution factor: $\left.1,3^{-1}, 3^{-2}, 3^{-3}, 3^{-4}, 3^{-5}\right)$ in serum-free DMEM culture medium were incubated for $2 \mathrm{~h}$ for virus absorption. After that, virus-containing medium was replaced with complete DMEM media and the cells were incubated for $24 \mathrm{~h}$. The next day, the cells were washed with PBS and fixed with $4 \%$ formaldehyde for $10 \mathrm{~min}$, followed by treating $0.1 \%$ Triton X-100 for permeabilization. After washing with 2\% PBA (2\% BSA in PBS solution), primary antibodies and secondary antibodies were treated in order. Mouse anti-cytomegalovirus monoclonal antibody (1:200, MAB810R Millipore, USA) was treated at room temperature for $1 \mathrm{~h}$. The cells were washed with 2\% PBA three times, and anti-mouse IgG-FITC antibody produced in goat (1:200, F0257, Sigma-Aldrich) was treated at room temperature for $1 \mathrm{~h}$. For nucleus staining, DAPI solution was treated for 10 min. Finally, fluorescence images were obtained by a Ti inverted fluorescence microscope equipped with a $4 \mathrm{x}$ objective IX 71 (Olympus, Tokyo, Japan) and a CoolSNAPcf charge coupled device camera (Photometrics, Tucson, AZ, USA) with Metamorph image analysis software (Molecular Devices, Sunnyvale, CA). The experiment was performed in triplicate.

RT-qPCR. The level of HCMV lytic replication and production was quantified through RT-qPCR by detecting the expression level of the viral transcripts of immediate early (IE2), early (UL44), and late (UL99) genes at 24 hours post infection (24 hpi), 48 hpi, 72 hpi. At first, HCMV-infected cells were harvested, and mRNAs were isolated from the cells with the TRIzol ${ }^{\mathrm{TM}}$ (Invitrogen) method. cDNAs were synthesized according to manufacturer's protocol (Invitrogen). Real-time qPCR was performed using the PowerUp ${ }^{\mathrm{TMT}} \mathrm{SYBR}^{\mathrm{TM}}$ Green Master Mix (applied biosystems) with the following primers. IE2 (forward primer); AACCCCGAGAAAGATGTCCT, IE2 (reverse primer): CCGGGGAGAGGAGTGTTAGT, UL44 (forward primer): CGTGTCGTGCTCCGTAACTA，UL44 (reverse primer): AGCTGGAATTCACGGCCAAT, UL99 (forward primer): CGGGGGAAACGACAGTAGTA, UL99 (reverse primer): CTGATGGTGGTGACGTTTTG. 


\begin{tabular}{|c|c|c|c|c|}
\hline Time of infection & $\mathbf{0 ~ h}$ & $\mathbf{2 4} \mathbf{~ h}$ & $\mathbf{4 8 ~ h}$ & $\mathbf{7 2} \mathbf{~ h}$ \\
\hline hcmv-miR-US25-1-5p & 693.94631 & 53088.614 & 124306.58 & 266216.23 \\
\hline hcmv-miR-UL112-3p & 8.2179362 & 844.65385 & 3994.3506 & 10513.985 \\
\hline hcmv-miR-US25-2-5p & 0.2988629 & 62.461786 & 178.05867 & 454.88772 \\
\hline hsa-miR-16-5p & 93125.36 & 89517.924 & 64555.012 & 88944.672 \\
\hline
\end{tabular}

Table S1. Argonaute-crosslinking and immunoprecipitation coupled to high-throughput sequencing (AGO-Clipseq) analysis of hcmv-miRNA expression profile after HCMV infection. 

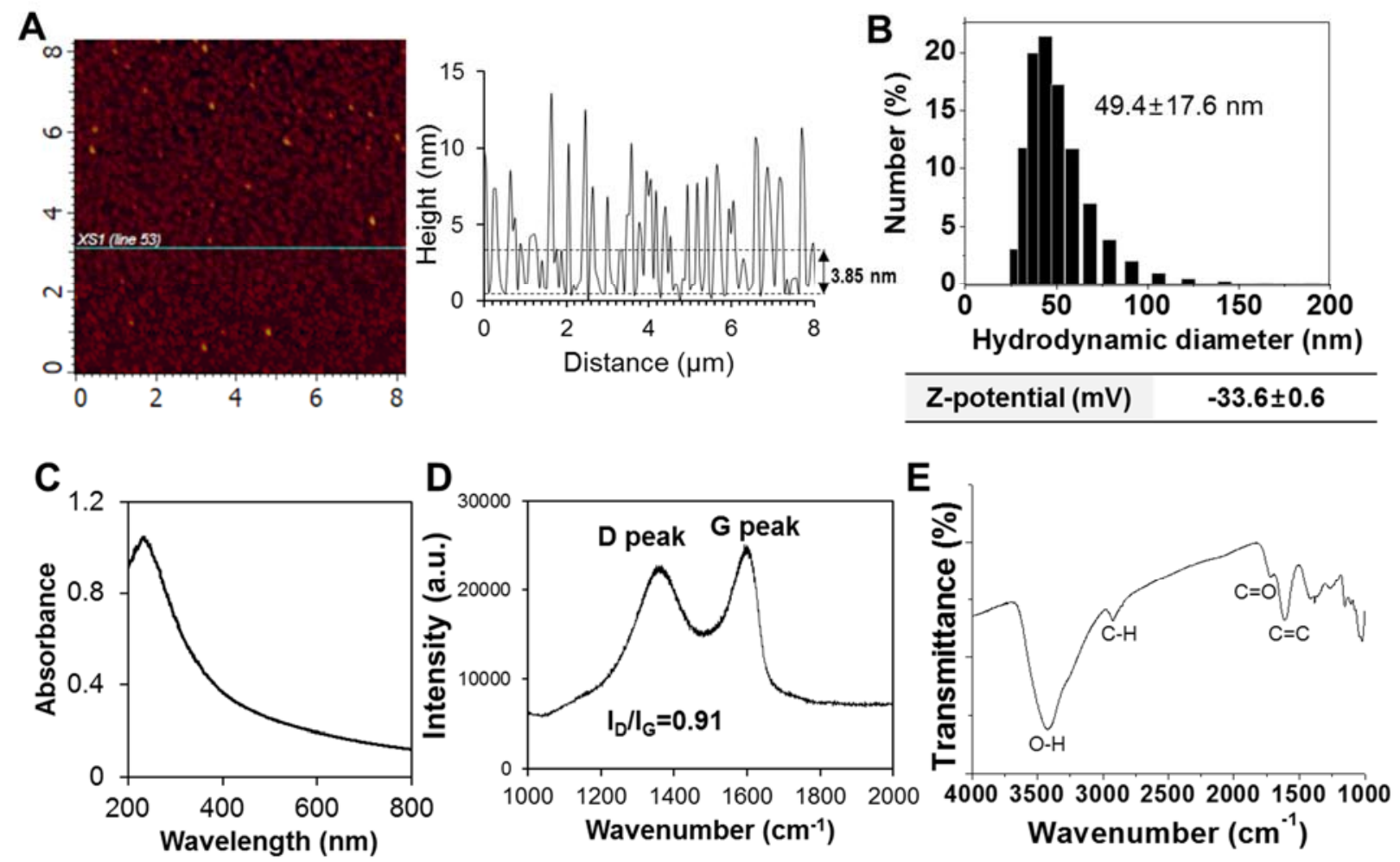

Figure S1. Characterization of the DGON. (A) AFM images with height profile. (B) Hydrodynamic diameter analyzed by dynamic light scattering (DLS), and zeta-potential (mV) analysis. (C) UV-Vis absorption spectra. (D) Raman spectra and its position of D and G peak including D/G peak intensity ratio $\left(I_{D} / I_{G}\right)$. (E) FT-IR spectra and assignment of the functional groups. 


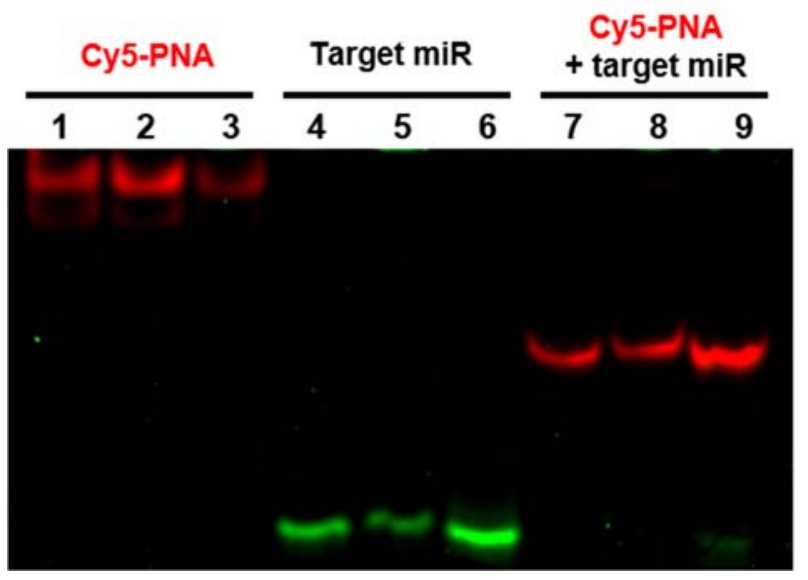

Figure S2. Non-denaturing polyacrylamide gel electrophoresis (15\%) to test the hybridization interaction between the Cy5-PNAs (10 pmol) and the target miRNAs (10 pmol). (lane 1: Cy5PNA1 (w/o target), lane 2: Cy5-PNA2 (w/o target), lane 3: Cy5-PNA3 (w/o target), lane 4: hcmv-miR-US25-1-5p, lane 5: hcmv-miR-UL112-3p, lane 6: hcmv-miR-US25-2-5p, lane 7: Cy5-PNA1 with target hemv-miR-US25-1-5p, lane 8: Cy5-PNA2 with target homv-miRUL112-3p, lane 9: Cy5-PNA3 with target homv-miR-US25-2-5p) 
A

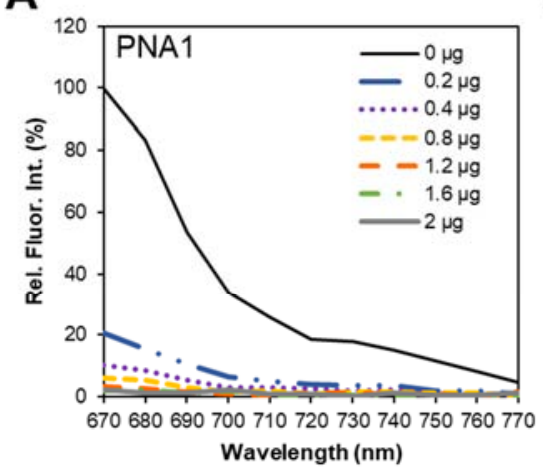

D

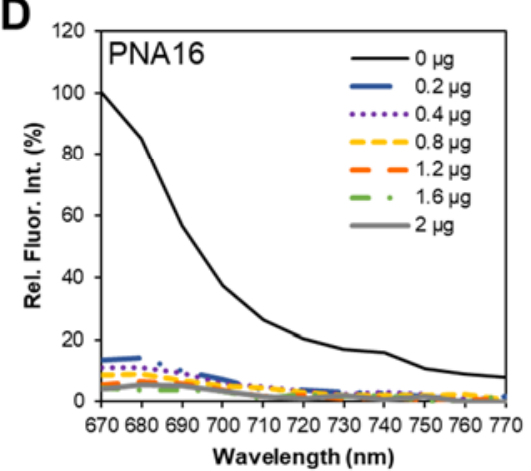

B

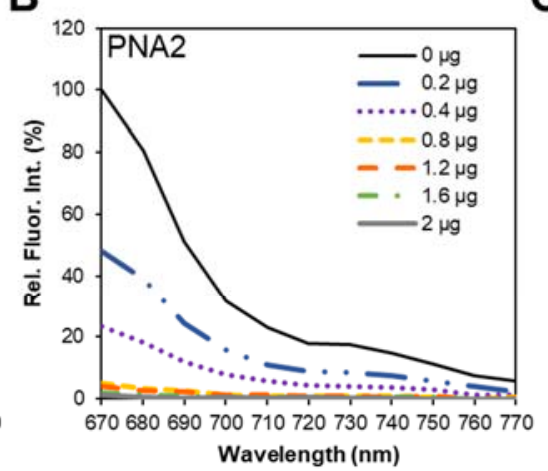

E

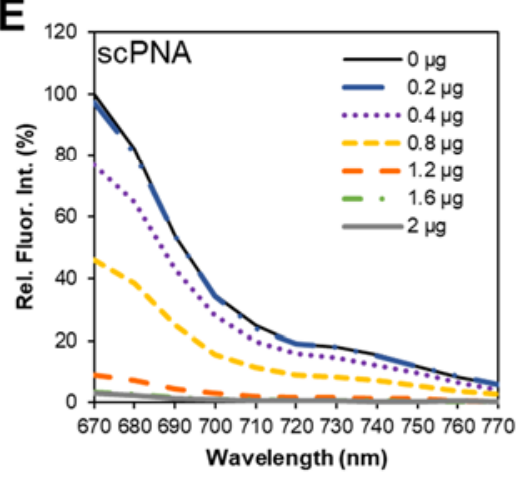

C

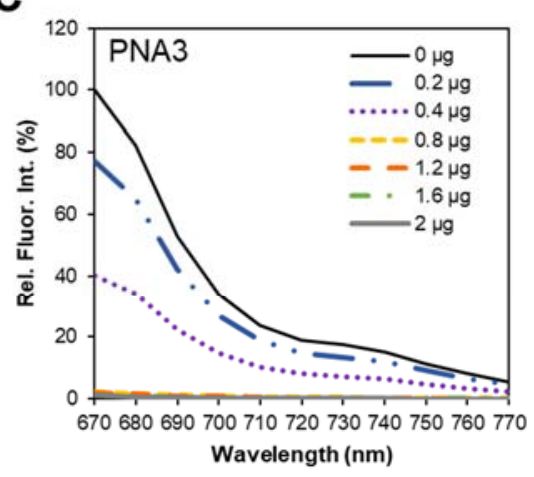

Figure S3. Fluorescence quenching test of each PNA probe (20 pmol) with DGON. Fluorescence spectra of (A) Cy5-PNA1, (B) Cy5-PNA2, (C) Cy5-PNA3, (D) Cy5-PNA16, (E) Cy5-scPNA with increasing amounts of DGON. The excitation/emission wavelengths $\left(\lambda_{e x} / \lambda_{e m}\right)$ of the fluorescence spectra were $647 / 670 \mathrm{~nm}$, respectively. 

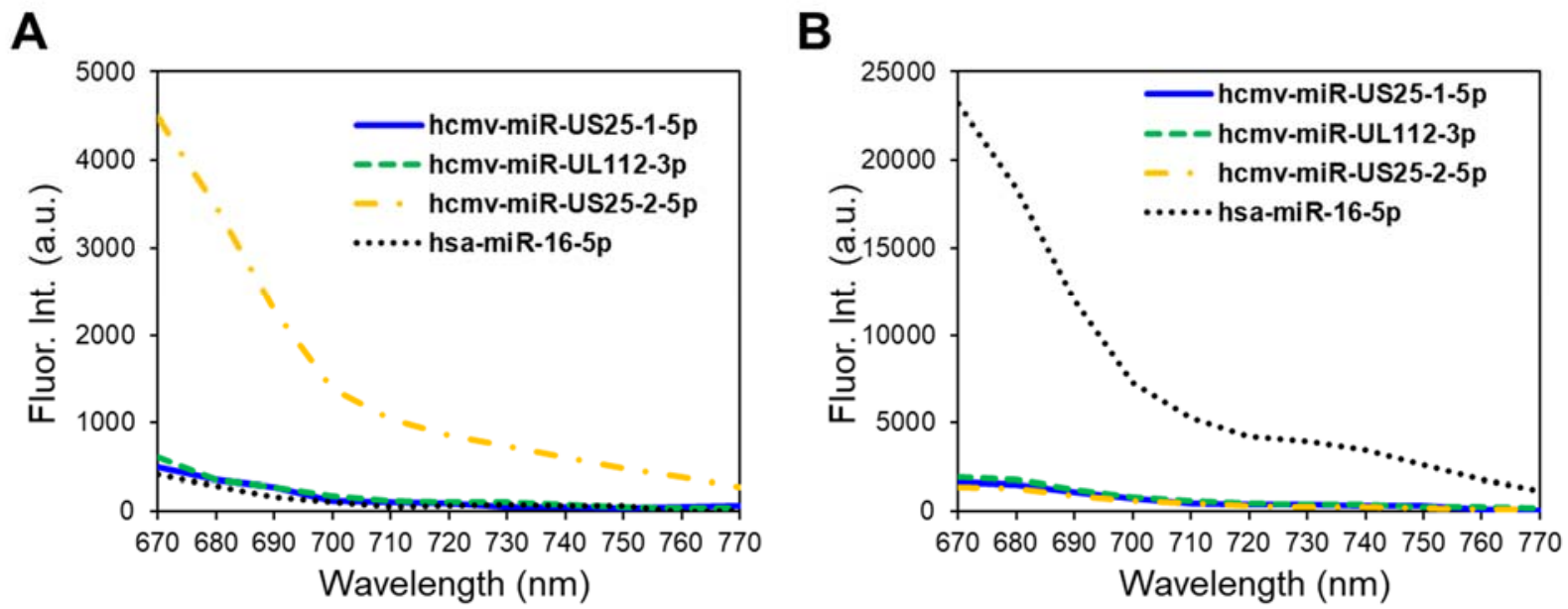

Figure S4. Specificity test of Cy5-PNA probes (PNA3, PNA16). Fluorescence emission spectra of (A) Cy5-PNA3/DGON and (B) Cy5-PNA16/DGON in the presence of homvmiR-US25-1-5p, hcmv-miR-UL112-3p, hcmv-US25-2-5p and hsa-miR-16-5p. The excitation/emission wavelengths $\left(\lambda_{e x} / \lambda_{e m}\right)$ of the fluorescence spectra were $647 / 670 \mathrm{~nm}$, respectively. 
A

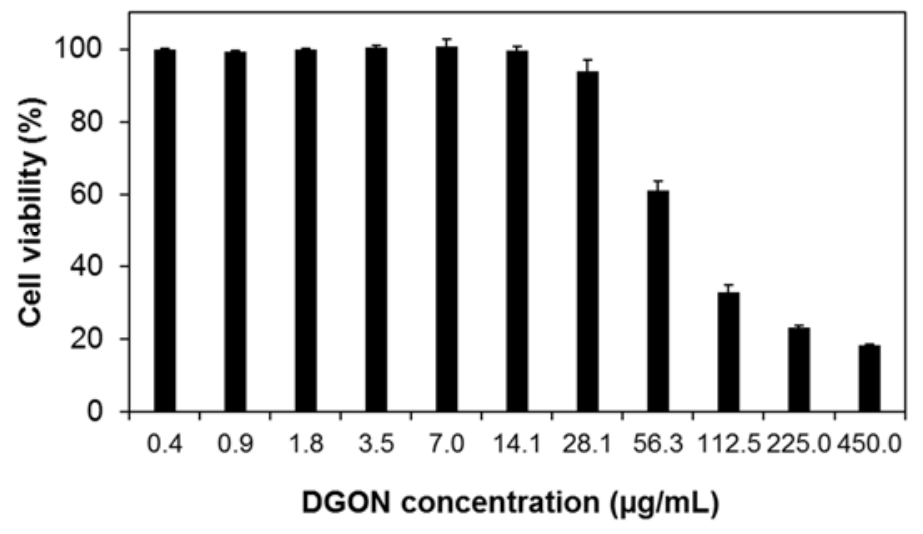

B

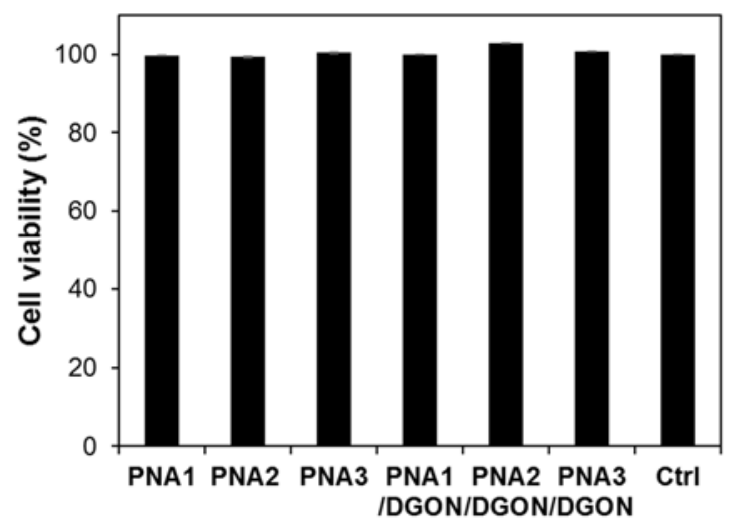

Figure S5. CCK-8 cell viability assay of HFF cells with (A) a wide concentration range of DGON (0-450 $\mu \mathrm{g} / \mathrm{mL})$, (B) PNA probes (20 pmol of each Cy5-PNA1, Cy5-PNA2, Cy5-PNA3) and each Cy5-PNA/DGON complex (Cy5-PNA1/DGON, Cy5-PNA2/DGON, Cy5PNA3/DGON, 20 pmol of each PNA, and DGON amount corresponding to each PNA). $2 \times$ $10^{4}$ cells were seeded per each well in 96 well plate, and each group of the materials (Total volume $100 \mu \mathrm{L}$ ) were incubated for $12 \mathrm{~h}$. The values are mean $\pm \mathrm{SEM}$ (standard error of the mean), (A) $n=3$, (B) $n=4$. 


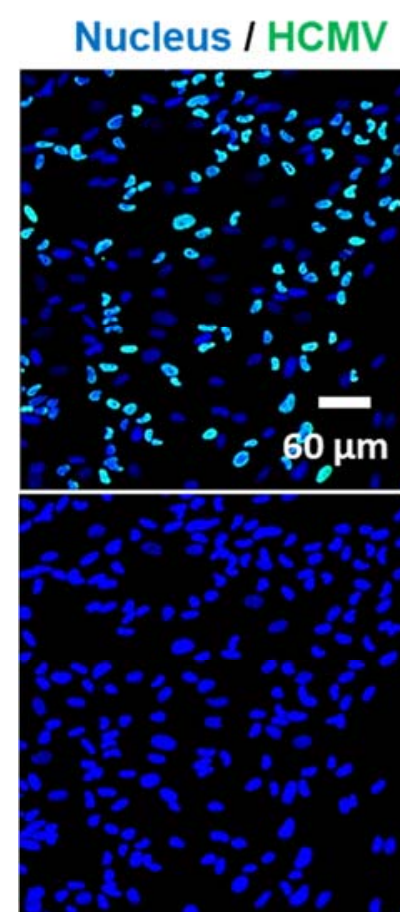

Nucleus
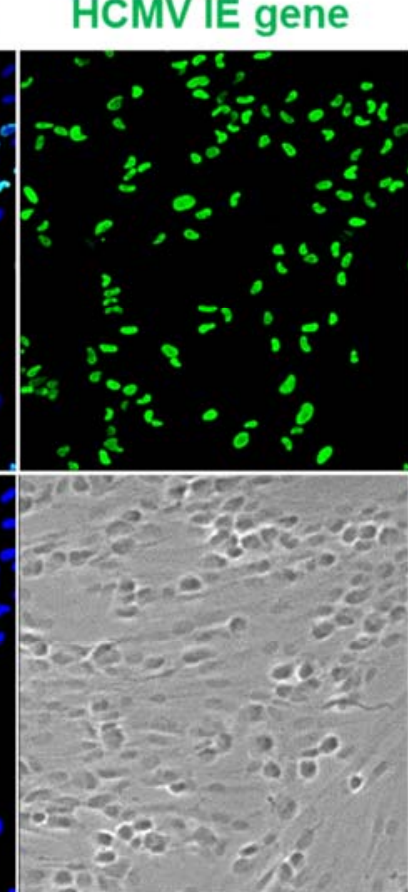

Bright field

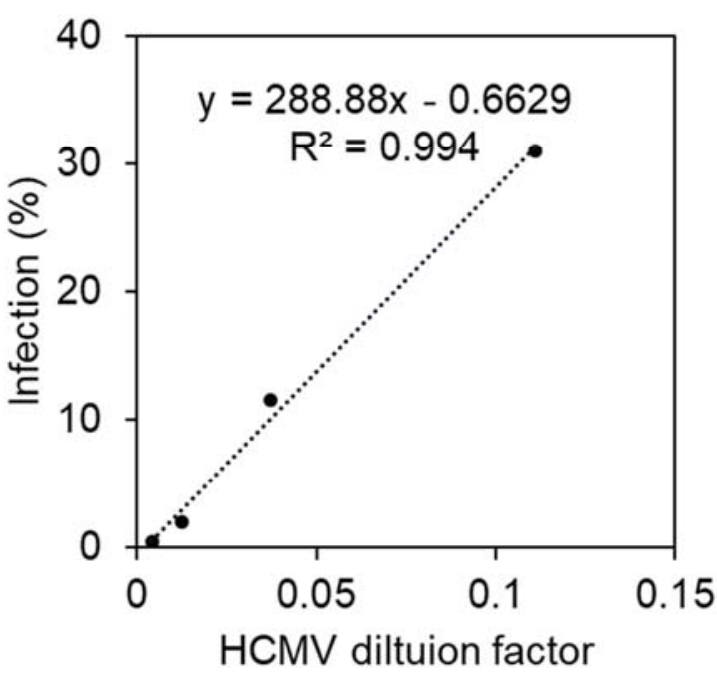

Figure S6. Immunofluorescence assay for titration of HCMV Towne ${ }_{\text {long }}$ strain after inoculation of the virus in HFF cells. Concentration of HCMV stock is calculated about $5.62 \times 10^{6} \mathrm{IFU} / \mathrm{mL}$. 
A

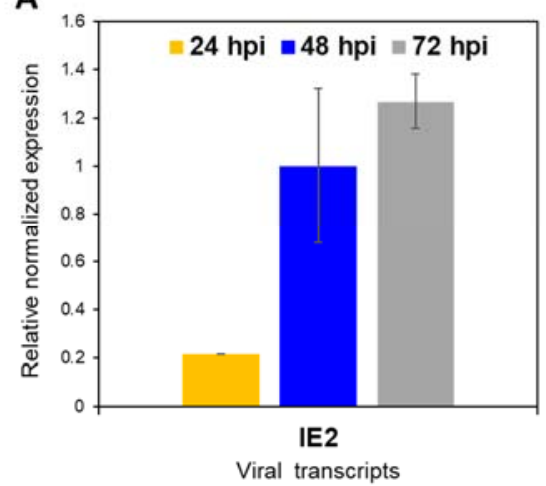

B

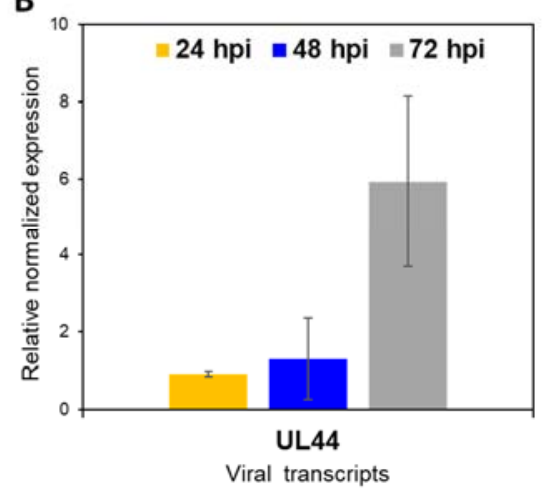

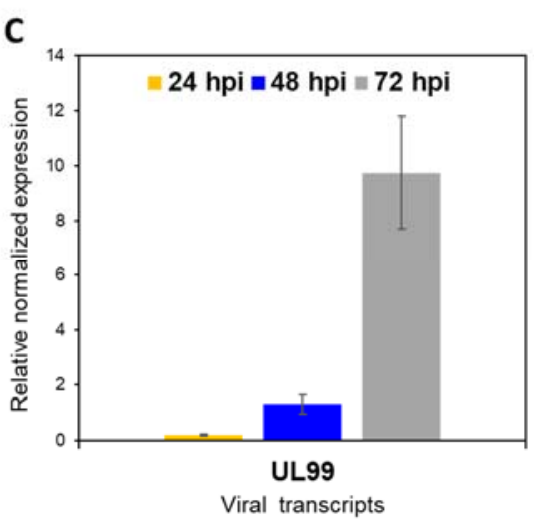

Figure S7. Analysis of the cycle of HCMV lytic replication and production through RT-qPCR by detecting the expression level of the viral mRNA transcripts for representative viral immediate early (IE2), early (UL44), and late (UL99) genes at 24 hours post infection (24 hpi), $48 \mathrm{hpi}, 72$ hpi. Each viral mRNA expression level was normalized against GAPDH mRNA. Data represent mean $\pm \mathrm{SEM}, n=3$ 


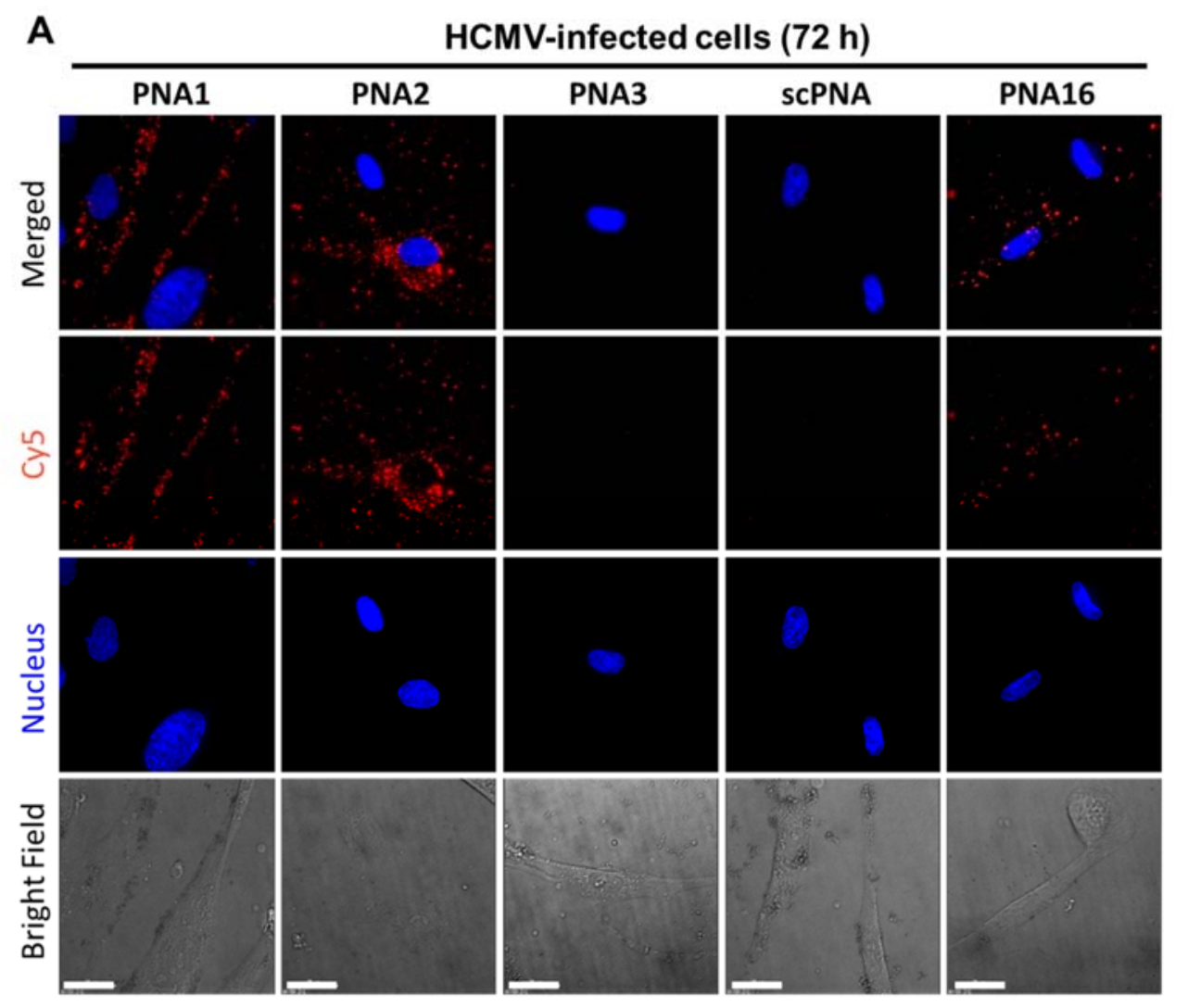

B

Uninfected cells

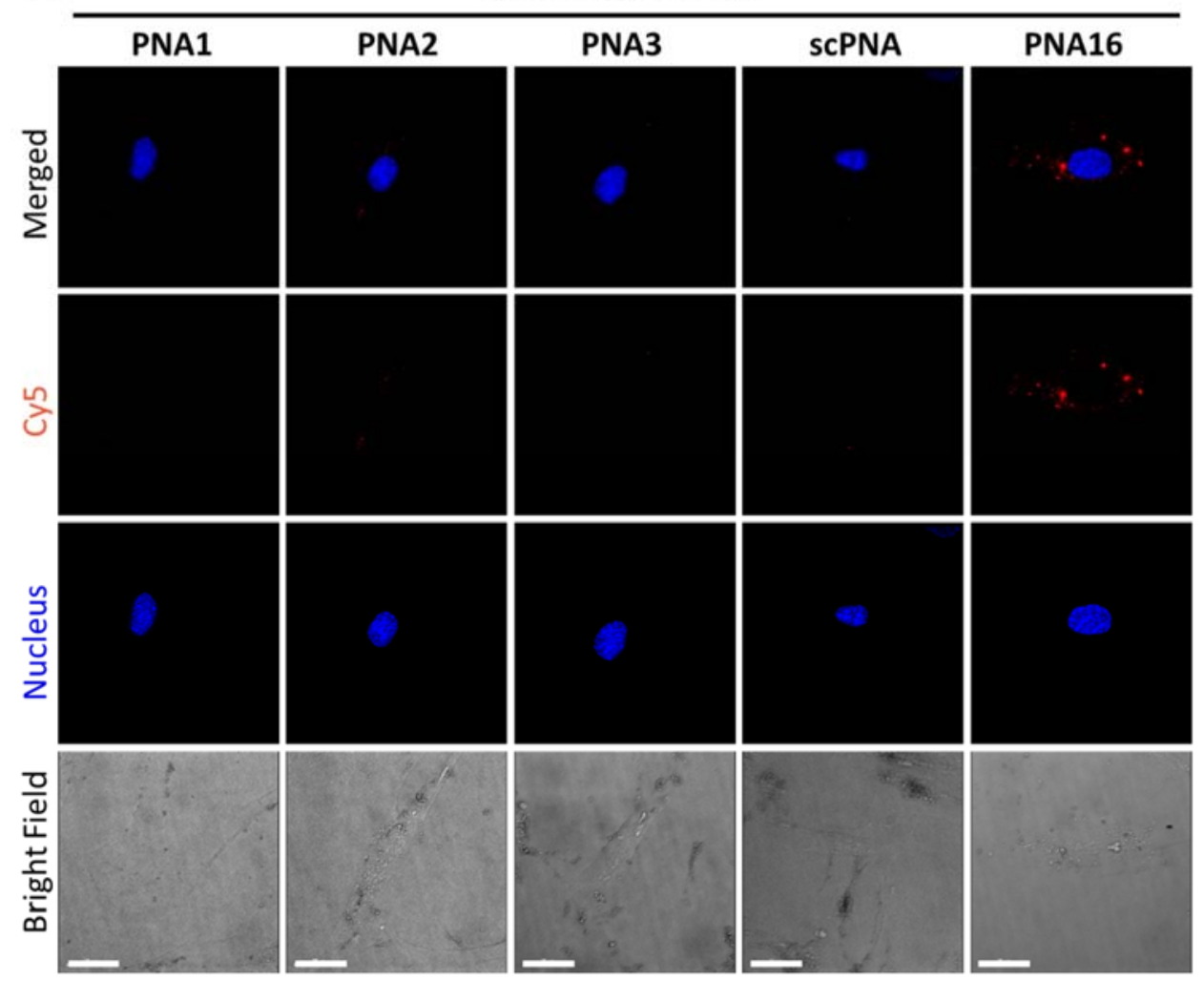

Figure S8. Fluorescence images of (A) HCMV-infected group (72 h) and (B) uninfected control group (ctrl grp) in HFF cells after treatment of the hcmv-miRNA sensors (Cy5PNA/DGON: PNA1, PNA2, PNA3, scPNA or PNA16 with each optimal amount of DGON) for $12 \mathrm{~h}$. The fluorescence images were obtained in living cells. The scale bar is $25 \mu \mathrm{m}$. (It is full version of the fluorescence images related to Figure 3). 

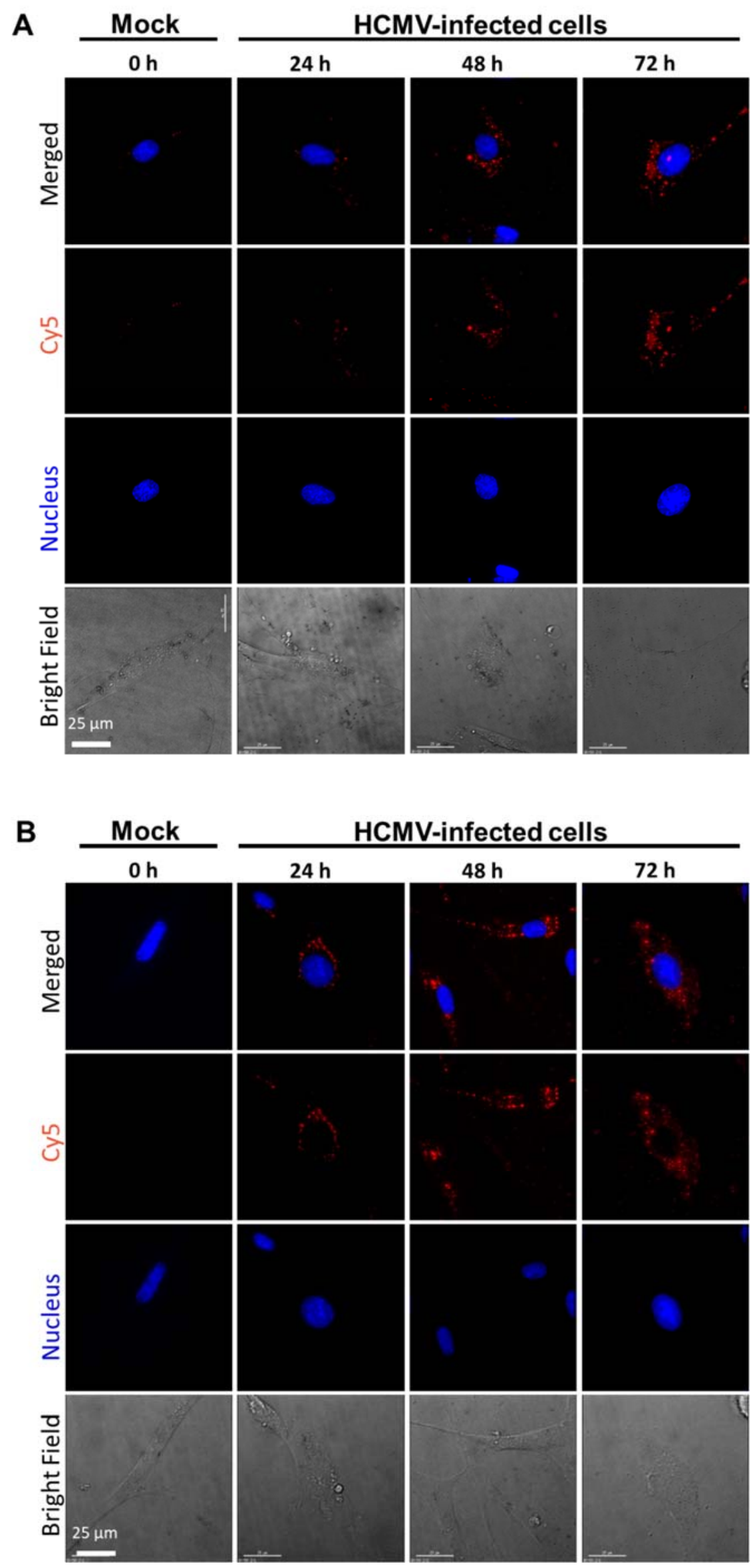

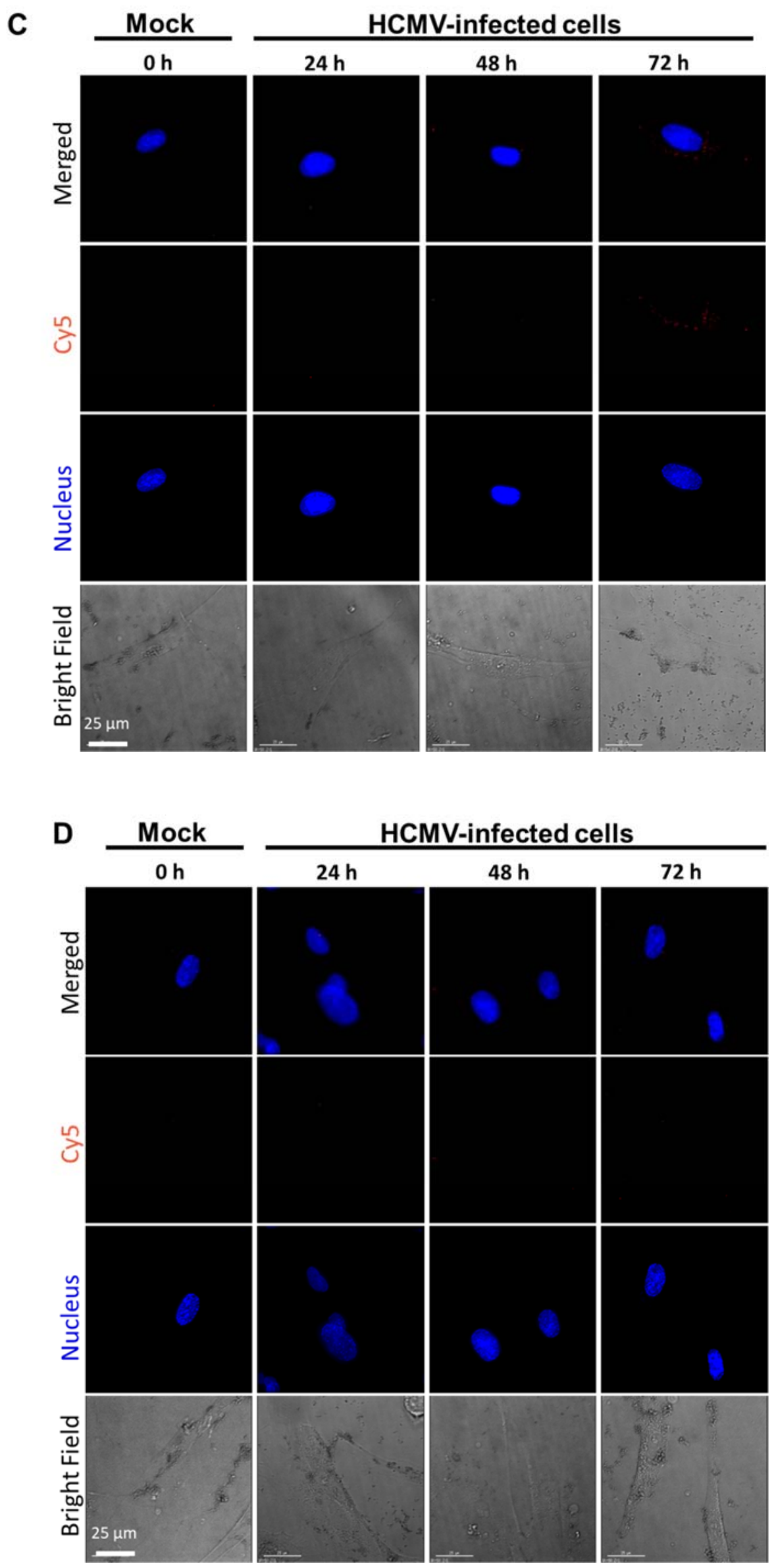


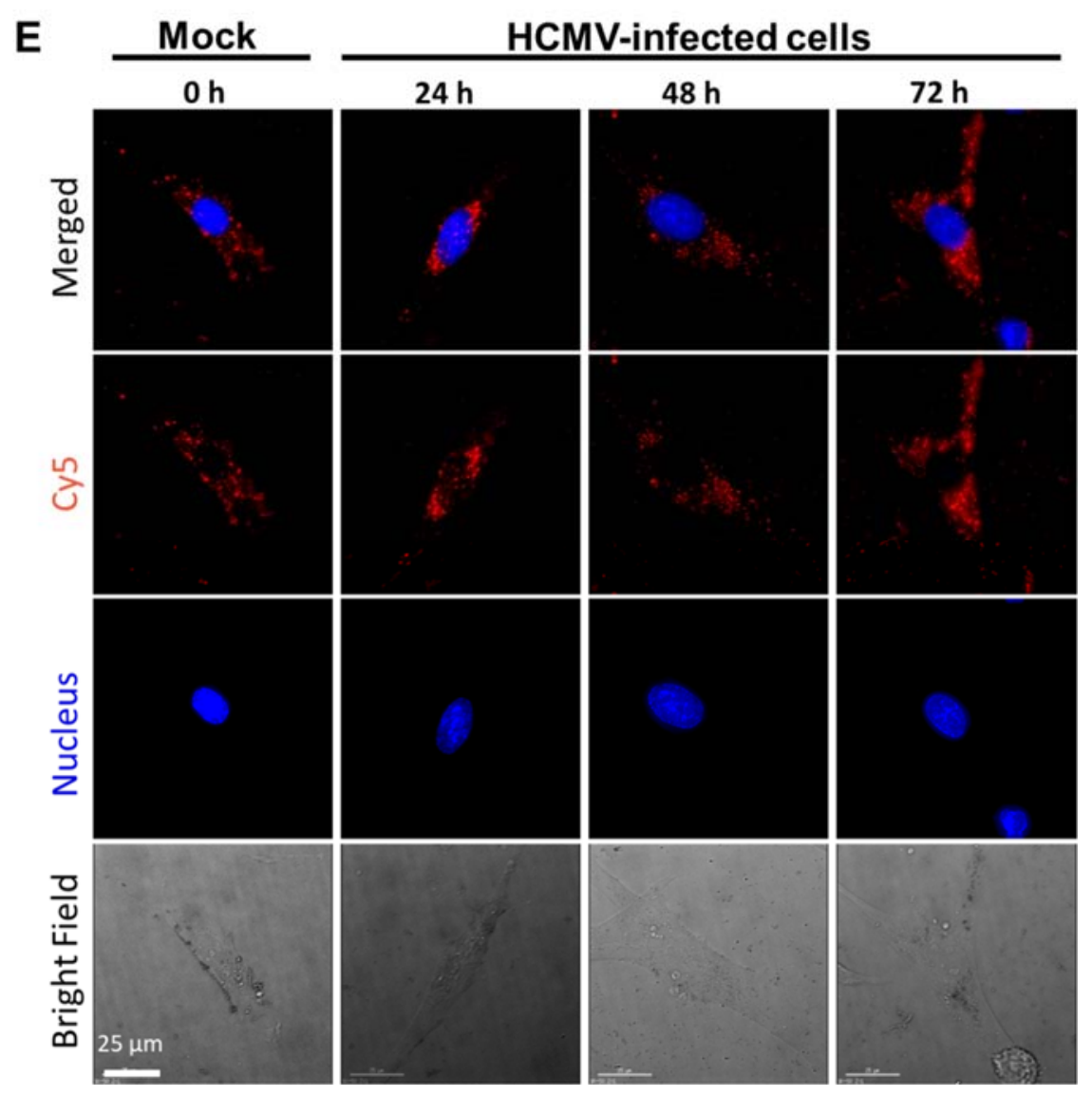

Figure S9. The fluorescence images of HCMV miRNA sensor, (A) Cy5-PNA1/DGON, (B) Cy5PNA2/DGON, (C) Cy5-PNA3/DGON, (D) Cy5-scPNA/DGON (a negative control group), (E) Cy5-PNA16/DGON (a positive control group) after time-course of HCMV infection (0 h (uninfected), $24 \mathrm{~h}, 48 \mathrm{~h}, 72 \mathrm{~h}$ ) in HFF cells. 20 pmol of each Cy5-PNA was treated for $12 \mathrm{~h}$ with optimal amount of DGON. The fluorescence images were obtained in living cells. The scale bar is $25 \mu \mathrm{m}$. (It is full version of the fluorescence images related to Figure 4C). 
A

Uninfected

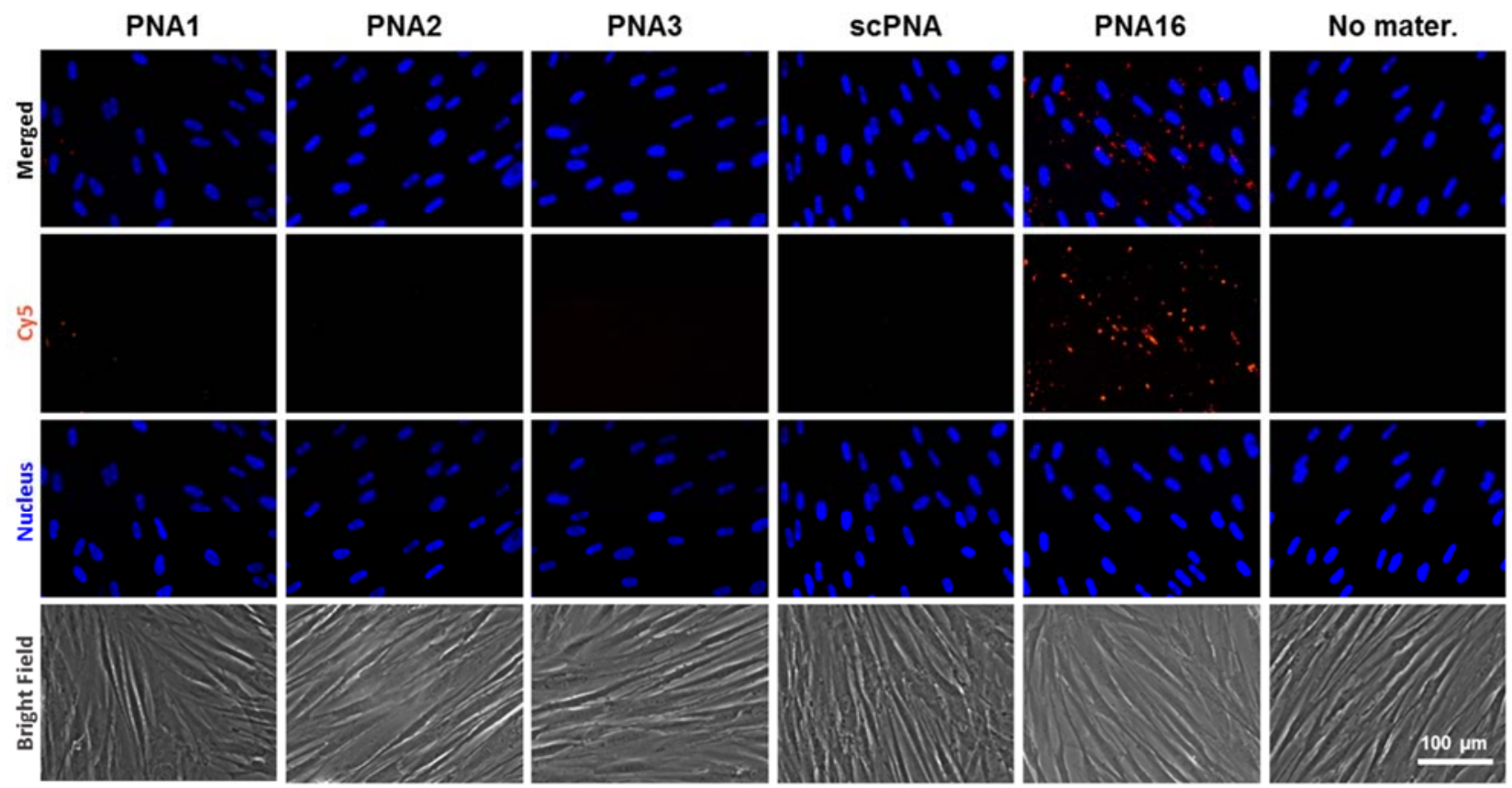

B

24 hpi

PNA1
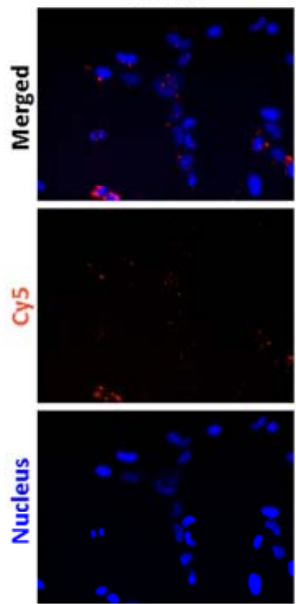

흔

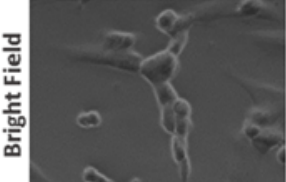

PNA2

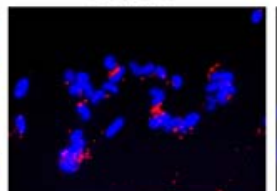

PNA3
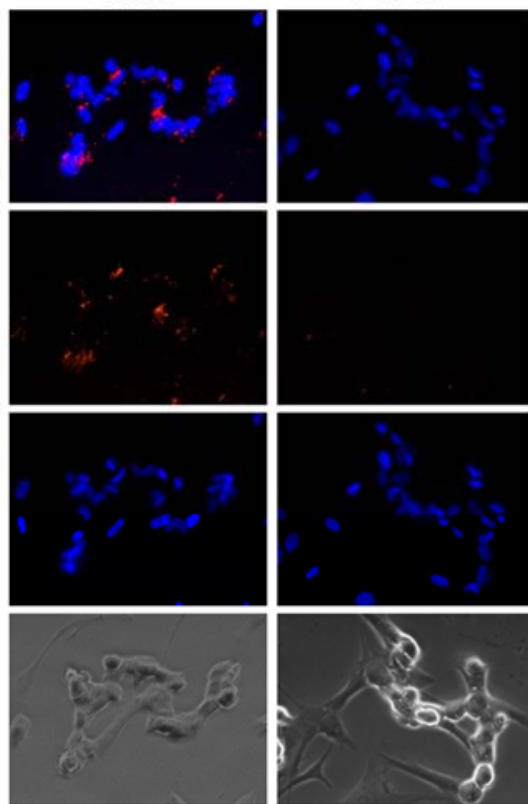

SCPNA

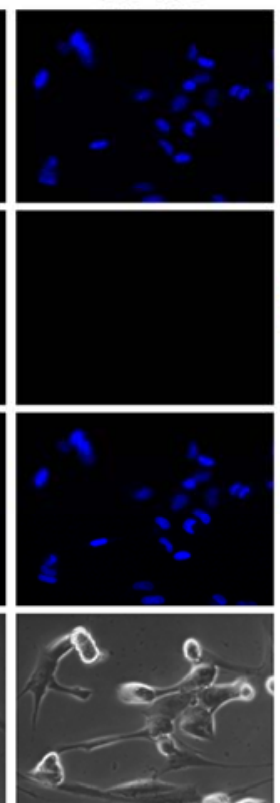

PNA16
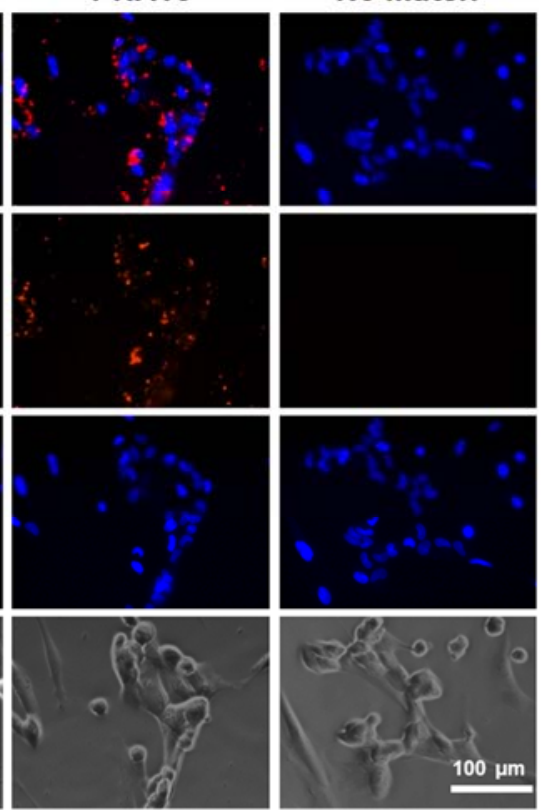
C

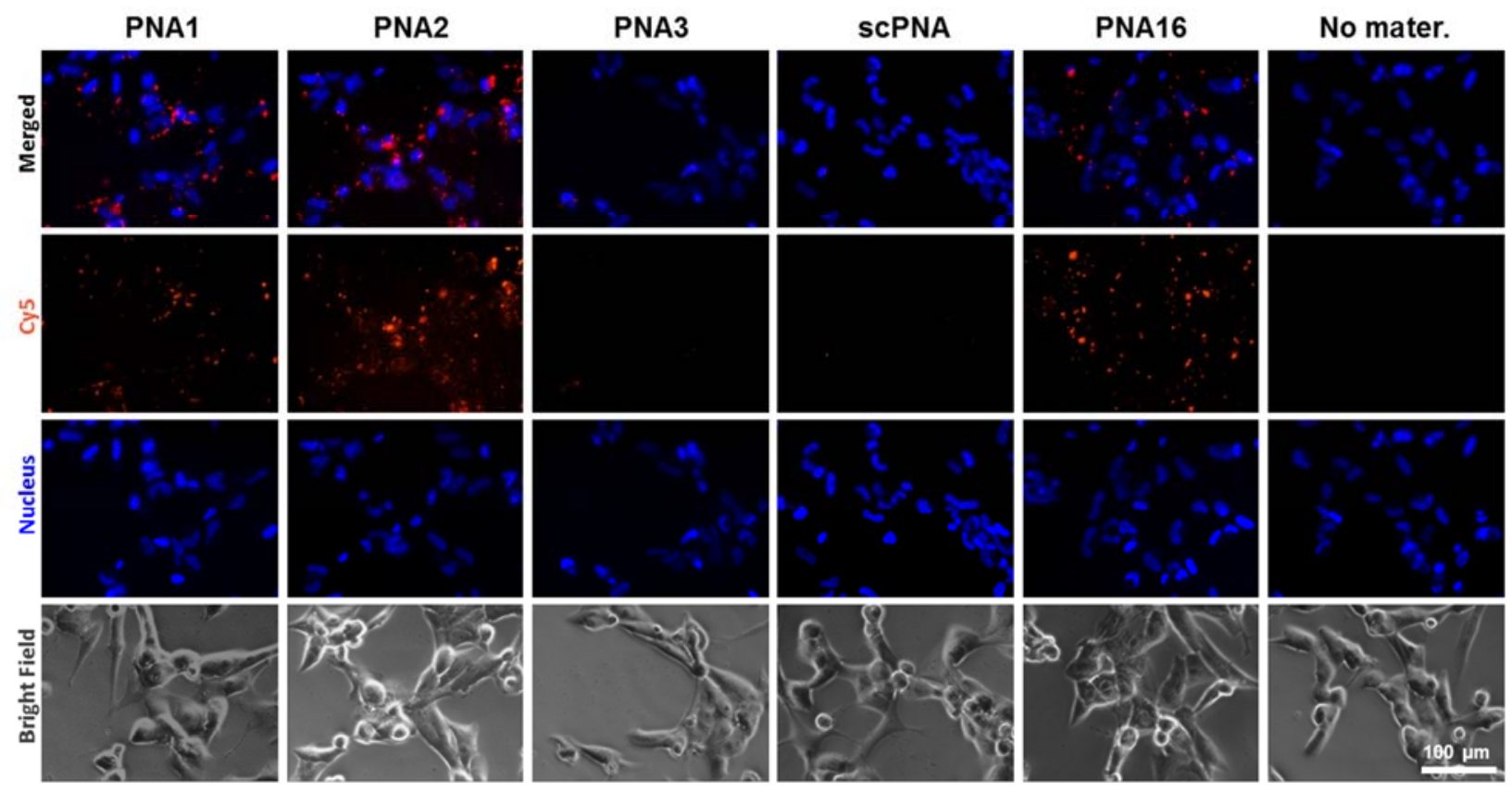

D
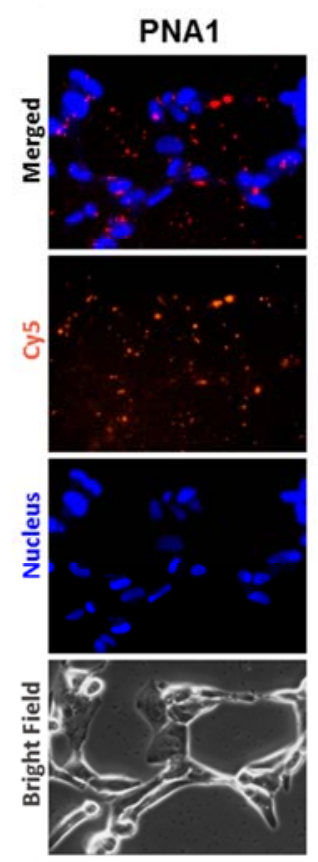

72 hpi
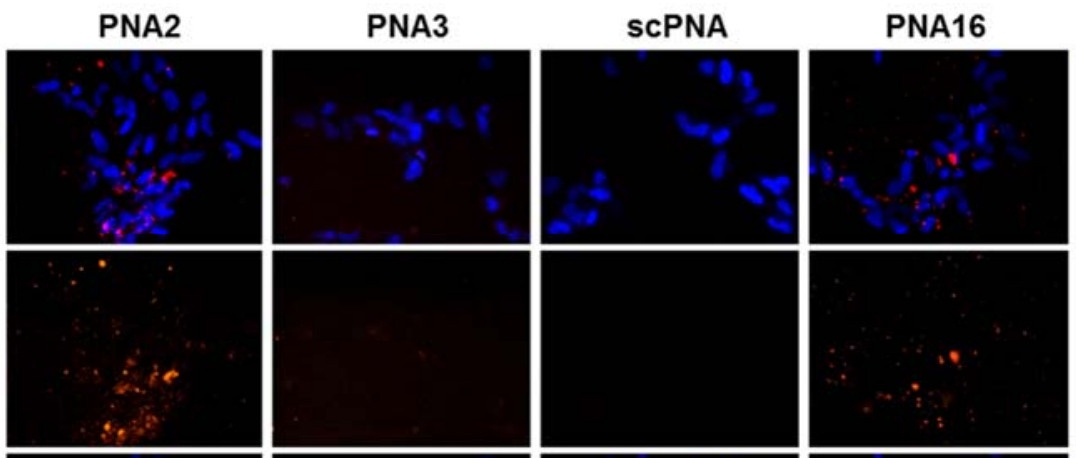

No mater.
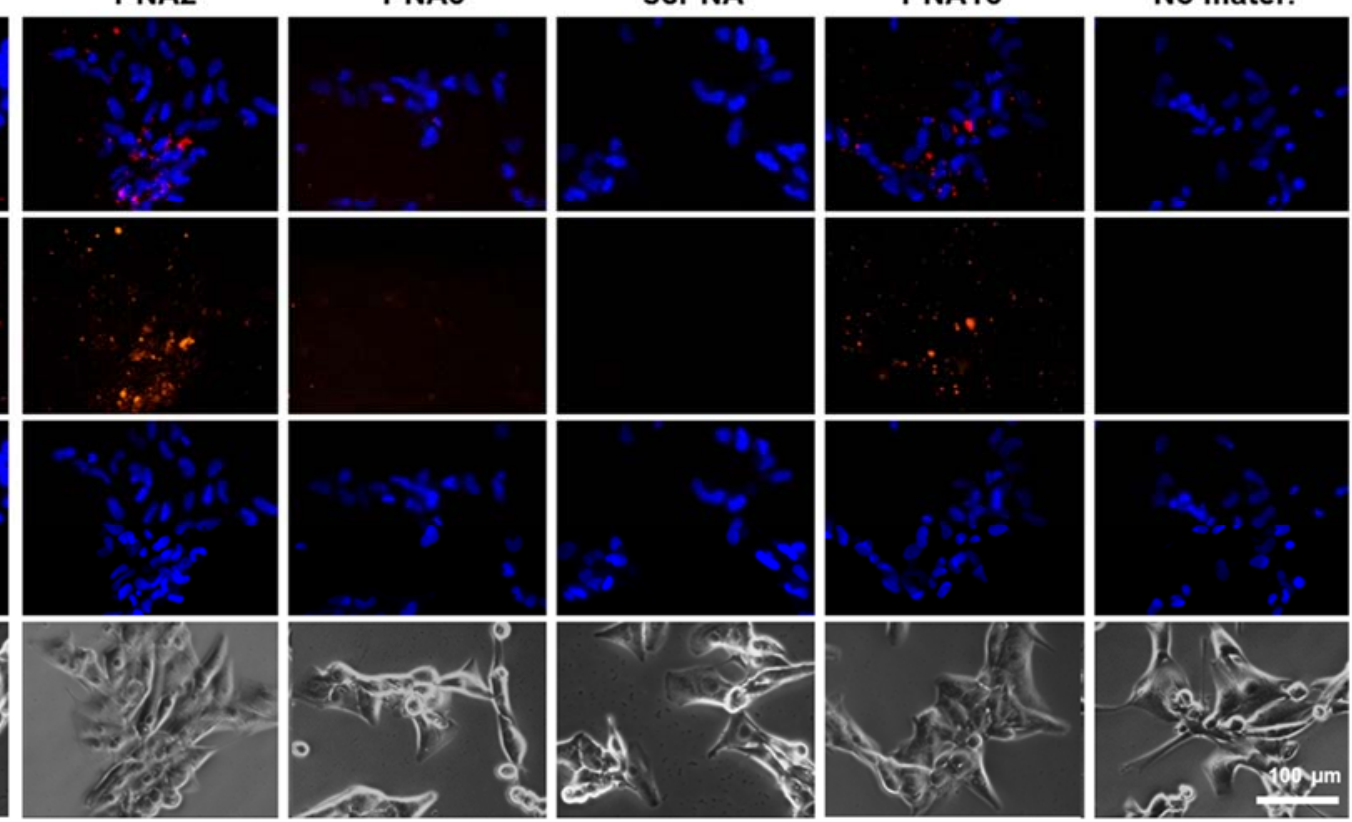

Figure S10. The fluorescence images of each HCMV miRNA sensor with a wider range. (A) uninfected. (B) 24 hpi (hours post infection). (C) 48 hpi. (D) 72 hpi. (It is supplementary data related to the Figure 4C). 